1 Myers G, Coyle D, Kowalski C, Srinivasan R. How can a young person wait over 90 hours in an emergency department for a bed? Psychiatr Bull 2014; 38: 250 .

Laxmi Kathuria is a consultant psychiatrist working at Yorkhill Hospital, Glasgow, UK, email: laxmi.kathuria@nnhs.net.

doi: $10.1192 / p b .39 .1 .50 a$

\section{It is more than just beds}

We read with interest the correspondence by Myers et al ${ }^{1}$ and echo their concern. In our region, child and adolescent psychiatrists are increasingly dealing with similar situations and are concerned for young people and their experience of services out of hours. We agree that there is no current system to find out bed availability and no external support to make this process efficient.

Fortunately, in our region we have an out-of-hours process whereby referrals can be made and we have agreement for two tier 4 providers to accept emergency admissions. Since this process was initiated, the referrals for out-of-hours beds have steadily increased and in the past 6 months 30 referrals were made, two-thirds of which were for people aged 17+. However, despite this process, only five young people were able to access these emergency beds in that period. The majority of young people had to wait until NHS England was available to manage the referral the next working day. Hence, there have also been calls in our region for daily bed state availability and for NHS England to be accessible out of hours.

Ensuring the best use of a scarce resource and the prioritisation of available beds requires high-quality and skilled clinical assessment. We also provide a gateway service/access assessment during working hours. This has averted the need for in-patient admission for a third of patients referred. It has been valued by referrers and ensures that the right patient accesses the right type of service. However, this service is not available out of hours.

We agree that increased bed provision is not the only solution. The divide in commissioning arrangements for tier 3 and tier 4 services means the development of alternatives to in-patient admission; outreach and crisis services and daypatient services have been patchy, too. In Birmingham we have developed a child and adolescent mental health home treatment service that has demonstrated a reduction in need for admission and cut length of stay by $50 \%$. Birmingham has also set up daytime and out-of-hours community emergency response and assessment teams that respond to emergency referrals from all the local general hospitals.

The report published by the Health Select Committee on 5 November 2014 highlights this major problem with access to in-patient services, as well as problems with commissioning and the lack of services which bridge the gap between inpatient and out-patient services. ${ }^{2}$ It takes a whole-systems view and recognises that the problem is about more than just beds.

1 Myers G, Coyle D, Kowalski C, Srinivasan R. How can a young person wait over 90 hours in an emergency department for a bed? Psychiatr Bull 2014; 38: 250.
2 House of Commons Health Committee. Children's and Adolescents' Mental Health and CAMHS. Third Report of Session 2014-15. TSO (The Stationery Office) (http://www.publications.parliament.uk/pa/ cm201415/cmselect/cmhealth/342/342.pdf).

Nicole K. Fung is a consultant child and adolescent psychiatrist at Heathlands Unit, email: nicole.fung@bch.nhs.uk, and Linda Cullen is clinical service director at Tier 4 CAMHS, Birmingham Children's Hospital NHS Foundation Trust, Parkview Clinic, Birmingham, UK.

doi: $10.1192 / p b .39 .1 .51$

\section{Self-diagnosing bipolar disorder: questions for clinicians}

It is not uncommon in psychiatry to receive referrals for patients who believe they have bipolar affective disorder. This has been explained partly by a trend of celebrities openly talking about having bipolar disorder along with an explosion of information about bipolar illness on the internet. ${ }^{1}$

We analysed the records of 46 patients who over a 3-year period requested referral to a community mental health team seeking a diagnosis of bipolar illness. The patients were predominantly young women (mean age 32 years, female:male ratio $31: 15)$. Clinically, they presented with problems of anxiety and low mood with a history of mood swings (90\%), racing thoughts (70\%), impulsivity (100\%) and overactivity (60\%). All patients had visited a website offering self-assessment for bipolar illness and reported scores being highly suggestive of a bipolar illness - this had influenced their decision to seek referral. Around $25 \%$ of patients reported seeing a TV programme featuring a celebrity talking about their bipolar illness. Five patients, of their own accord, had joined their local Bipolar UK support group before the assessment.

None of the patients were given the diagnosis of bipolar illness at initial assessment. All were given formulations about their problems in terms of mood swings, coping and lifestyle issues. The ICD-10 diagnostic categories were mixed anxiety depression/adjustment disorder/dysthymia (20 patients); emotionally unstable personality disorder (10); alcoholism/ alcohol misuse (5); no psychiatric diagnosis (11). About a third of patients, after having their history taken, readily agreed at the end of the first meeting that they were not suffering from a bipolar illness. Five patients asked for a second opinion; all were experiencing relationship problems.

Our experience highlights the issues that may be encountered while assessing patients who actively seek diagnosis of a bipolar illness. There is merit in taking the patient into confidence about the confusion surrounding diagnosing bipolar illness and the risks associated with medical treatment. Also, while trying to arrive at a diagnosis, it may be best to look for classical or severe bipolar illness and if the evidence is not strongly suggestive then the diagnosis should be avoided or deferred until conclusive evidence is obtained.

1 Chan D, Sireling L. 'I want to be bipolar' ... a new phenomenon. Psychiatrist 2010; 34:103-5.

Avneet Sharma is a consultant psychiatrist with South Gloucestershire Recovery Team, Blackberry Hill Hospital, Bristol, UK, email: avneet. sharma@nhs.net, and Jitender Kumar is a speciality trainee (ST5) in forensic psychiatry at Fulbourne Hospital, Cambridge.

doi: 10.1192/pb.39.1.51a 